\title{
Bearing the mark
}

Arabinosyl nucleosides are an addition to the toolkit of DNA-labeling methods.

It was not long ago that radioactive nucleoside analogs were the common approach to label replicating DNA in cells and tissues.

Radioactive labels were later replaced by more user-friendly synthetic nucleoside analogs such as bromodeoxyuridine (BrdU). Although antibodies to BrdU could be used to detect the incorporated thymidine ana$\log$ and tag cells that were actively replicating their DNA, this staining requires harsh treatments to denature the cells' DNA as well as fixation. Moreover, antibodies have poor tissue penetration, and BrdU itself is both toxic and mutagenic when applied in high concentrations.

Small organic dyes are compatible with deep-tissue imaging, and labeling can be done using click reactions that do not require sample fixation or DNA denaturation. A strategy for chemical DNA labeling using the deoxythymidine analog 5-ethynyl-2' deoxyuridine (EdU) offered great possibilities for DNA labeling in vitro and in vivo. EdU has gained much popularity and has been extensively used in cell-proliferation and differentiation and many other studies over the last couple of years.

Despite its many advantages, EdU is more toxic than BrdU, hindering its application for long-term labeling studies and perpetuating the search for improved DNA labeling tags.

Anne Neef and Nathan Luedtke at the University of Zurich are pursuing the search for new synthetic compounds with interesting physical properties, including DNA binding. In recent work, they tested nucleoside derivatives with a $\mathrm{D}$-arabinose configuration as alternative or complementary DNA labels to EdU and BrdU.

They synthesized a small family of 5-ethynyl-arabino-uridine derivatives and tested their capacity to label genomic DNA as well as their toxic activities in diverse cell types. The group found that several of these compounds - in particular (2'S)-2'-deoxy2 'fluoro-5-ethynyluridine (F-ara-EdU)effectively labeled DNA at concentrations that produced little or no cellular toxicity. F-ara-EdU proved advantageous for pulsechase experiments aimed at birth-dating DNA in vivo. Feeding the analog to zebrafish embryos in combination with BrdU, Neef and Luedtke identified quiescent and senescent cells during the embryos' development.

Arabinosyl nucleosides may prove an interesting addition to the toolkit of DNA labeling methods, with just the right balance of detection sensitivity and low cellular toxicity to the cells.

\section{Erika Pastrana}

\section{RESEARCH PAPERS}

Neef, A.B. \& Luedtke, N.W. Dynamic metabolic labeling of DNA in vivo with arabinosyl nucleosides. Proc. Natl. Acad. Sci. USA 108, 20404-20409 (2011). 